\title{
Causalismo e interpretacionismo en la explicación psicológica: el caso del psicoanálisis
}

Ricardo Braun

Universidad de Lima

Lima, Perú

Desde un punto de vista filosófico se discute el modelo psicoanalítico como forma de explicación científica. Como una extensión de la psicología popular, el psicoanálisis postula la existencia de creencias y deseos, y explica la conducta humana como interacción causal entre las creencias y deseos inconscientes. Como ciencia médica, el psicoanálisis debería estar comprometido con una visión realista de la etiología de las neurosis. Sin embargo, se argumenta que, al carecer el psicoanálisis de una teoría de la verdad, la recolección de datos y la posterior interpretación invitan a la arbitrariedad. En consecuencia, las interpretaciones tienen un valor meramente instrumental con miras a los resultados terapéuticos. El psicoanálisis, se concluye, conduce a una visión no realista de la psicología popular.

psicología popular / explicación / causalidad / interpretación

\section{Causalism and interpretationism in psychological explanation: the case of psychoanalysis}

From a philosophical standpoint, the psychoanalytic model as a form of scientific explanation is discussed. As an extension of folk psychology, psychoanalysis posits the existence of beliefs and desires, and explains human behavior as a result of the causal interaction of unconscious beliefs and desires. If, as it is argued, psychoanalysis is a medical science, it ought to be committed to a realistic view of the etiology of neuroses. However, due to the lack of a theory of truth, the recollection of data and their further interpretation are beset with arbitrariness. Thus, interpretations have an instrumental value for therapeutic outcome. In sum, psychoanalysis is not committed to a realistic view of folk psychology.

folk psychology / explanation / causality / interpretation


En un trabajo anterior ${ }^{1}$, he presentado y discutido de una manera general, algunos de los problemas que suscita una visión no causal del interpretacionismo en la explicación psicológica. Mi objetivo fue demostrar que el interpretacionismo no es incompatible con el causalismo. Mi propósito en este trabajo es analizar filosóficamente el caso particular del psicoanálisis. El psicoanálisis, como teoría y terapia del comportamiento humano depende considerablemente de los efectos de la interpretación. Puede, por lo tanto, constituir una buena ilustración de cómo la interpretación funciona en la explicación de la conducta. Pero, como espero demostrar, existen razones poderosas para dudar de que el psicoanálisis, como actividad interpretativa, pueda generar explicaciones científicas adecuadas.

Lo que motiva este artículo es el hecho de que el psicoanálisis puede ser visto como una extensión de la psicología popular (folk psychology en el vocabulario corriente de la filosofía angloamericana $)^{2}$. En efecto, el psi-

1 "La explicación científica y el caso de la psicología”, investigación inédita. Código 5001C1996003, Dirección Universitaria Coordinadora de Investigación Científica, DUCIC, Universidad de Lima.

2 Para una introducción al tema de la psicología popular en español ver Broncano (1995). "El término 'Psicología Popular' denota así un cuerpo de explicaciones y predicciones de la conducta que se caracteriza esencialmente por su apelación a estados mentales con contenido - deseos, creencias, miedos, intensiones, intuiciones y, en gene- coanálisis postula la existencia de deseos y creencias de una forma análoga a la que encontramos en la psicología popular. Pero, como intento mostrar, el psicoanálisis no está comprometido con una visión realista de la psicología popular. Al contrario, debido a su singular método y la ambigüedad de su significado, el psicoanálisis no proporciona explicaciones causales.

No voy a concentrarme en el análisis exegético de los trabajos de Freud sino en los argumentos que subyacen a su trabajo y a aquél de los practicantes actuales del psicoanálisis. Mucho de lo que se ha discutido en filosofía concerniente a la validez y condición científica del psicoanálisis se ha limitado a la exégesis de los escritos de Freud. Pero esto es problemático, puesto que no es sólo comúnmente sabido que Freud cambió sus puntos de vista acerca del psicoanálisis varias veces y aún mantuvo posiciones contradictorias, sino que simplemente podría estar equivocado en algunas de sus conclusiones. Tomar a Freud como la medida de lo que es correcto o incorrecto en el psicoanálisis, es argumentar falazmente por ape-

\footnotetext{
ral, estados psicológicos construidos en términos proposicionales- como causas de tal conducta. Es una explicación típica de la Psicología Popular la de que Pedro llegó tarde a su cita con Manuel porque creía que habían quedado a una hora diferente. Es tal creencia la que, de acuerdo con la Psicología Popular, constituye la causa interna de la conducta de nuestro amigo" (Broncano, 1995, p. 246).
} 
lación a la autoridad, usando las creencias de Freud como evidencia en vez de evaluar la fuerza de los argumentos en los que su posición estaba basada.

Antes de discutir los problemas de la explicación psicoanalítica, en la primera sección, proporciono lo que considero es el núcleo del psicoanálisis: las interacciones clínicas. En la segunda sección describo los elementos del psicoanálisis, considerado como una ciencia clínica. La tercera sección está dedicada al análisis de la recolección de datos. Finalmente, en la cuarta sección, discuto cómo los datos son interpretados. Mi objetivo es mostrar que el psicoanálisis no proporciona una adecuada explicación causal. He preferido concentrarme en aquellos argumentos que promueven una visión causal de la explicación. Aparte de una breve alusión, no discuto la posición hermenéutica a la que me he dedicado en un trabajo anterior. En la discusión, incorporo a continuación ciertas evidencias empíricas. Estoy convencido de que mucho de nuestra discusión filosófica es inútil si dejamos de reconocer algunas sugerencias de los estudios empíricos. Esto es particularmente cierto en las discusiones sobre el psicoanálisis en las que tradicionalmente se ha evadido la confrontación en la arena empírica.

\section{EL NÚCLEO DEL PSICOANÁLISIS}

Uno de los problemas que uno enfrenta al evaluar el psicoanálisis es que el psicoanálisis no es un cuerpo monolítico de conocimiento. Al contrario, si algo ha caracterizado la historia del psicoanálisis desde su creación hasta nuestros días, es la constante controversia tanto de teóricos como de usuarios. Y, sin embargo, debería haber un núcleo fundamental de creencias que pueden ser identificadas y distinguidas de otros enfoques psicológicos. Se debe tener en cuenta que la evaluación de la situación del psicoanálisis dependerá de las presuposiciones que se tenga del núcleo de contenido del psicoanálisis. Por ello, es importante mantener una visión lo más general posible sobre los conceptos y prácticas que caracterizan el psicoanálisis de hoy, para evitar mostrar una facción particular como representativa del conjunto completo de creencias que constituye el psicoanálisis.

En un congreso reciente (Roma, 1989), el tema más importante de la agenda era la preocupación sobre el futuro del psicoanálisis. Los psicoanalistas presentes se preguntaban si emergerían nuevas divisiones (o disoluciones), o si habría una reunificación gradual y una reintegración de la inmensa diversidad y pluralidad ${ }^{3}$. Lo

3 Véase la Conferencia Presidencial de Robert Wallerstein en el Congreso Internacional de Psicoanálisis en Roma, 1989, en Wallerstein (1990). 
que parece incontrovertible es que los diferentes sistemas teóricos psicoanalíticos -de Klein, egopsicológico, objeto-relacional, por mencionar algunos- tienen una implicancia en el marco explicativo y la práctica clínica. $\mathrm{Y}$, sin embargo, las personas que trabajan bajo esos sistemas se llaman a sí mismas psicoanalistas. En medio de tal diversidad, es apropiado preguntarse por los cimientos de lo que propiamente pertenece al reino del psicoanálisis. Puesto que no ayuda tomar la postura de "todo vale" o que cualquier cosa que tenga que ver con lo mental pueda ser construido como psicoanálisis.

En su trabajo "¿Un psicoanálisis o muchos?", Wallerstein sugería que el camino para salir de esta paradoja era virar de la diversidad de lealtades teoréticas al común denominador del trabajo clínico (Wallerstein, 1988). Ciertamente, esta posición está confirmada con los influyentes trabajos de psicoanalistas como Shafer (1976), Klein (1976) y otros que han abandonado mucho de lo más ortodoxo de la metapsicología freudiana y se han concentrado en las interacciones clínicas. De acuerdo con esta visión, el psi-

Anteriormente, Wallerstein (1988) había mostrado su preocupación por el mismo asunto. Cf. también Rangell (1988). coanálisis debe limitarse a las formulaciones de los conceptos derivados directamente de las confrontaciones y experiencias con pacientes, la llamada "teoría clínica de la experiencia cercana" (Klein, 1976). Por más novedosa que parezca esta postura, se parece mucho a la visión del núcleo del psicoanálisis que tenía Freud, tal como lo cuenta en su historia del psicoanálisis: “Toda línea de investigación que reconozca estos dos hechos (transferencia y resistencia) y los toma como el punto de partida de su trabajo tiene el derecho de llamarse psicoanálisis" (O.C. XIV, p. 16) ${ }^{4}$. La dinámica de la transferencia (y contratransferencia) y resistencia es uno de los ingredientes fundamentales del ambiente clínico en cualquier forma de psicoanálisis. Y esto no debería ser sorpresa. Desde sus comienzos, el psicoanálisis ha estado directamente relacionado con la práctica clínica. Aparte de algunos ejemplos que Freud sacó de su propia experien$\mathrm{cia}^{5}$, el psicoanálisis fue pensado para - y también fue resultado del- trabajo

4 Utilizaré la abreviación O.C. (obras completas) para referirme a la reconocida edición inglesa (Standard Edition) del Institute of Psycho-Analysis.

5 El locus classicus es el recuento del mismo Freud y la interpretación del llamado "sueño de Irma" (O.C., IV, pp.106-121). 
clínico $^{6}$. Aun cuando se reconocía las consecuencias de la teoría psicoanalítica en muchos campos de la investigación, en 1913 Freud resumía el contenido del psicoanálisis de esta manera: "El psico-análisis es un procedimiento médico que busca la cura de ciertas formas de enfermedad nerviosa (las neurosis) a través de una técnica psicológica" (O.C., XIII, p. 165).

Aun asumiendo, por hipótesis, que el común denominador del psicoanálisis es el conjunto de interacciones clínicas en los consultorios, no se puede decir que haya un consenso en la teoría que subyace a estas interacciones. Algunos consideran que a pesar de lo común de estas interacciones, es en el entorno clínico en el que "aparecen las profundas y potencialmente irreconciliables controversias" (Wallerstein, 1990, p. 8). Pero, a pesar de todas las discrepancias, el psicoanálisis todavía utiliza (con diferentes grados de énfasis) algunas de las técnicas tradicionales diseñadas por el mismo Freud, a saber: el uso de sueños, la asociación libre y la dinámica de la transferencia y contratransferencia. Como se dijo en el mencionado congreso, los psicoanalistas

6 Una minoría todavía considera que el psicoanálisis es una psicología general, es decir, aplicable a todo el sistema de la personalidad, a las interacciones interpersonales y a las capacidades psicológicas. Para una crítica de esta posición ver Edelson (1988, Parte I). aún creen que este conjunto de técnicas proporciona el medio para la recolección de datos, y para traer el alivio a los disturbios psíquicos que adolecen sus pacientes ${ }^{7}$. El énfasis puesto en el trabajo clínico y no en la empresa teórica ha devenido en una disociación entre la teoría y la técnica derivada, a tal punto que algunos practicantes ni siquiera endosan teoría alguna. Muchos psicoanalistas actuales prefieren reconocer una ignorancia teórica y prefieren admitir una posición más pragmática: "No soy un teórico, soy un clínico en ejercicio" (Wallerstein, 1990, p.12).

La discusión que sigue toma en consideración el núcleo del psicoanálisis como el presentado ${ }^{8}$. Por lo tanto, el análisis debe enfocarse en el éxito del psicoanálisis como una ciencia clínica, lo que se traduce en la evaluación de la profundidad de las explicaciones de la etiología del comportamiento patológi-

7 Wallerstein comparó los informes presentados por tres psicoanalistas que provenían de diferentes perspectivas teoréticas. Fundamentalmente, encuentra que las técnicas básicas mencionadas son usadas por los tres analistas. El problema, como veremos, no es el uso de la técnica, sino cómo los datos pueden ser recolectados cuando hay tantas discrepancias al detalle que producen, literalmente, diferentes datos. Para una perspectiva similar acerca de técnicas comunes ver Miller (1988, p. 666) y Eagle (1983, pp. 44-45).

8 He tratado de poner las diferentes visiones del psicoanálisis bajo un común denominador, pero, como veremos luego, estas diferencias profundizarán aún más los problemas del psicoanálisis como una explicación psicológica. 
co y la eficacia de sus terapias. Esto deja fuera la evaluación de la metapsicología que, para algunos analistas practicantes, es superflua y dañina ${ }^{9}$.

\section{El PSICOANÁlisis COMO CIENCIA CLÍNICA}

Aquellos que escogen la identificación del psicoanálisis como una ciencia clínica hacen un compromiso con un modelo causal de explicación. En un modelo médico, las personas enfermas son el foco de la investigación y el objetivo fundamental es la identificación de las causas que produce una enfermedad particular. El segundo objetivo es la reducción o supresión de la dolencia del paciente a través de una terapia apropiada. La identificación de las causas, presumiblemente, debería dar alguna luz sobre los posibles caminos hacia la cura de la enfermedad. Pero no es así siempre.

En efecto, el conocimiento de las causas de una enfermedad no se refleja necesariamente en el resultado positivo de la terapia. Es posible identificar las causas y ser incapaz de hacer mucho para curar la enfermedad, como ocurre en

9 Metapsicología, de acuerdo a Freud, es la descripción que emplea los aspectos dinámicos, topográficos y económicos del proceso psíquico. En otras palabras, en una descripción metapsicológica hay tres conceptos psicológicos que entran en juego: fuerzas, sistemas y energía, cada uno de acuerdo a sus respectivos aspectos. Véase Edelson (1988, p. 114) y Macmillan (1997, pp. 524ss). nuestros días con la epidemia del sida. Tenemos razones poderosas para aceptar la etiología del sida y, sin embargo, no tenemos una cura efectiva contra el mal. Pero en el caso del psicoanálisis, el método y los conceptos que ocurren en el entorno clínico están fundamentalmente dirigidos a obtener un resultado terapéutico positivo. El que Freud se hubiera decepcionado con los resultados de su terapia y disminuyera su énfasis en el tratamiento médico, es otro asunto ${ }^{10}$. Aún más, algunas veces en la historia de la medicina no hay una relación necesaria entre la teoría y la práctica terapéutica. Pero en el psicoanálisis, uno esperaría que la evidencia del resultado terapéutico diera alguna luz a la esfera teórica, e, inversamente, la metodología de recolección de datos -es decir, la búsqueda de causas- debería ser parte de la cura ${ }^{11}$.

Independientemente de la persuasión teorética, los psicoanalistas creen que la acción terapéutica es el resultado de la interpretación, en otras palabras, el

10 La decepción de Freud se puede leer en sus últimos escritos en los que prefiere llamarse "analista" a lo que en su trabajo temprano llamó "doctor" (Nash, 1989, p.329).

11 Freud escribe lo siguiente: “... el paciente sufre de una suerte de ignorancia, y si uno remueve esa ignorancia dándole información (acerca de la conexión causal entre la enfermedad y su vida, acerca de su experiencia en la infancia, y así sucesivamente) debiera recuperarse. El factor patológico no es la ignorancia en sí misma, pero la raiz de la ignorancia son sus resistencias inter- 
objetivo de la interpretación es producir cambio (Edelson, 1988, p. 78 y Schwaber, 1990, p. 229). Pero, ¿qué es lo que hay que interpretar y cómo la interpretación puede tener impacto en la cura de la enfermedad?

Si reducimos el núcleo del psicoanálisis al entorno clínico, entonces, los síntomas neuróticos y sus síntomas acompañantes tales como los sueños y actos fallidos, deben ser el fenómeno principal que se quiere explicar, si hay algo que explicar.

Las explicaciones de los síntomas neuróticos pueden ser pensadas como una extensión de nuestra psicología popular puesto que se explica utilizando entidades tales como la creencia y el deseo. La extensión ocurre porque se introducen creencias y deseos no conscientes que son olvidados por la psicología popular. Análogamente a la psicología popular, el psicoanálisis explica ciertos comportamientos como resultado de las interacciones -o mayormente conflictos- de creencias y deseos. Pero, a diferencia de la psicología popular, que descansa en pre-

nas; son ellas las que produjeron la ignorancia, y la mantienen. La tarea del tratamiento consiste en combatir esas resistencias. Informando al paciente de aquello que no conoce porque to ha reprimido es uno de los preliminares del tratamiento" (O.C., XI, p. 225). Debe anotarse, sin embargo, que Freud no garantizaba la remoción de la patología con el solo hecho de proporcionar información causal de los patógenos de la aflicción. suposiciones racionales del comportamiento, los deseos y creencias no conscientes no operan en una forma ordenada, esto es, usando un silogismo práctico (Wollheim, 1993, p. 95). Los deseos y creencias no conscientes operan de una forma que origina lo que Freud llamó "vacío de conciencia" (O.C., I, pp. 228-229). Precisamente son estos vacíos inexplicables lo que constituye el dominio del psicoanálisis.

El psicoanálisis, como la psicología popular, asume que los estados mentales tienen contenido, pero algunos contenidos son inexplicables porque no parecen tener una conexión causal con otro contenido o contenidos a los que el sujeto tiene acceso consciente. El trabajo del psicoanálisis es mostrar que la discontinuidad en la conexión causal entre los contenidos causales es debida, o bien a los conflictos o dilemas, o bien a los esfuerzos del agente para resolver esos conflictos o dilemas. De acuerdo con el psicoanálisis clásico, los conflictos o dilemas son el resultado de los deseos múltiples, que compiten al mismo tiempo y en el mismo momento por su satisfacción. El problema es que algunos de esos deseos son incompatibles -mutuamente exclusivos- o los recursos -organísmicos o ambientalesson limitados para su satisfacción. Un individuo pretenderá resolver estos conflictos y dilemas, pero es improbable que su esfuerzo sea totalmente exitoso. En tanto el problema esté bajo control del estado consciente, habrá 
conexión causal, aun cuando traiga frustración o miseria. Todo esto es parte del comportamiento normal.

Pero hay otros conflictos y dilemas que, al fracasar el esfuerzo de solución consciente, son eliminados de la esfera consciente y pasan a ser inaccesibles a la conciencia. Esos problemas están allí y puesto que no son conscientes, no pueden ser objeto de una evaluación de contenido -para una solución posterior- por parte del agente. La lucha por la solución continúa pero a nivel fuera de la conciencia. La persona no sabe por qué se comporta o se siente como lo hace, puesto que las conexiones causales de la vida diaria no son las mismas que se usan para pensar y planificar. Allí es cuando la persona se encuentra con los vacíos de conciencia. La miseria -el comportamiento neurótico- es el precio que se paga por la incapacidad de encontrar una solución adecuada.

El tratamiento del comportamiento neurótico involucra explicar los vacíos causales en la vida mental consciente del paciente. En otras palabras, el objetivo del psicoanalista es hacer inteligible las creencias y deseos que el paciente encuentra incomprensible. Y la manera de hacerse consciente -insight en la jerga psicoanalítica- es comprendiendo los contenidos de esas creencias y deseos. Aquí es donde la intepretación tiene lugar. De acuerdo con el método psicoanalítico -tan general como pueda pensarse- el analista trata de establecer o asignar significado a los datos recibidos. A través de ello, los conflictos y dilemas inconscientes son traídos a la conciencia y ese ejercicio supone un efecto terapéutico (O.C., $\mathrm{X}$, pp. 120-121) ${ }^{12}$. Veamos cómo funciona la interpretación psicoanalítica.

\section{LOS DATOS DE LA INTERPRETACIÓN}

El método básico del psicoanálisis para recolectar datos es la asociación libre. Se le pide al paciente que asocie libremente cualquier palabra o idea que se le viene a la mente. El paciente, sin control ni inhibición, permite que las asociaciones fluyan libremente $y$, con la ayuda del analista, alcanza la meta de comprender el significado de su comportamiento. Puesto que Freud era un determinista, vio la cadena de asociaciones no como algo azaroso, sino como los pasos naturales hacia el inconsciente (Orbach, 1995, p. 41).

En un modelo médico, los datos son esenciales para la comprensión de la enfermedad. Aparte de cualquier consideración de eficacia terapéutica, prácticas médicas estándares, tales como la diagnosis y la posible prognosis, dependen de la identificación de las

12 Freud nota, sin embargo, que no hay garantía de éxito con cada esfuerzo interpretativo: "El éxito terapéutico, sin embargo, no es nuestra meta principal; buscamos, más bien, permitir al paciente obtener un alcance de los deseos inconscientes" (ibíd.). Pero parece un ejercicio ocioso para el paciente si no se va a obtener algún beneficio terapéutico. 
causas de la enfermedad. Análogamente, uno esperaría cierto grado de confiabilidad en la recolección de datos en el entorno psicoanalítico, puesto que los datos son la ruta hacia la causa de la enfermedad mental. Pero, como espero demostrar, éste no parece ser el caso en el psicoanálisis.

Antes de discutir algunos problemas de la recolección de datos, quisiera expresar mis dudas acerca de los muy conocidos argumentos de Adolf Grünbaum que cuestionan la validez de los datos provenientes de las asociaciones libres (Grünbaum, 1984). A pesar de las acusaciones de inexactitudes históricas de sus trabajos (Nash, 1989; Richardson, 1990; Sachs, 1991; Levy, 1996), Grünbaum ha insistido recientemente en que "la atribución del éxito terapéutico a la remoción de las represiones no sólo fue, sino es hasta nuestros días, el solo sustento de la supuesta habilidad de las asociaciones libres de los pacientes para certificar las causas" (1992, p. 24). Quiero tocar dos puntos aquí. Mucho de lo que Grünbaum sostiene se basa en su lectura de Freud ${ }^{13}$. Esto no es algo que

13 Grünbaum sostiene correctamente que Freud defendió la relación entre éxito terapéutico e identificación de los presumibles patógenos de las neurosis utilizando la asociación libre, pero no en todos los casos. De la evidencia del éxito terapéutico, en algunos casos, Freud generalizó la confiabilidad del método (Grünbaum,1992, p.25). quiero comentar en este trabajo, aunque los estudiosos de Freud sostienen que no hay consistencia en su pensamiento. Pero, como mencioné anteriormente, el desafío de Grünbaum acerca de la validación de las asociaciones libres debería estar dirigido al mismo método, como un método de diagnosis. Los efectos terapéuticos son deseables, pero no deberían depender necesariamente de las asociaciones. Freud podría haber creado un método brillante para descubrir el inconsciente e identificar patógenos, y, al mismo tiempo, ser incapaz de encontrar la cura para la aflicción. Creo que el desafío al psicoanálisis tiene que proceder de forma diferente.

Los argumentos que utiliza Freud para validar la creencia de que los datos obtenidos de la asociación libre conducirá hacia los síntomas neuróticos son, en el mejor de los casos, una opinión de un clínico. Y desde el tiempo de Freud ha habido pocos estudios que hayan revisado la objetividad y validez de la asociación libre (Macmillan, 1997, pp. 566-568) ${ }^{14}$. Consideremos lo que Freud sostiene en sus Conferencias introductorias sobre psicoanálisis cuando se refiere a los

14 Hasta este momento estoy concentrándome en la asociación libre como método para recolectar datos, y no en su interpretación, que discuto posteriormente. 
datos de la asociación libre producto de un sueño:

La primera cosa que le ocurre al soñador está destinada a traer aquello que estamos buscando. Es un hecho que eso es lo que le ha ocurrido a ese hombre y nada más... puede ser probado que la idea producida por el hombre no era arbitraria ni indeterminada ni desconectada con lo que estamos buscando (O.C., XVI, p. 106).

Pero, desafortunadamente, no hay tal prueba. ¿Cómo sabemos que lo que vino a la mente del paciente está causalmente conectado con el inconsciente? Queda claro del pasaje la creencia que tenía Freud en el determinismo. Recurrir al determinismo lleva a la infalibilidad, puesto que cualquier cosa que el paciente diga puede ser tomado como prueba de una inferencia causal. Sin embargo, intuitivamente, uno podría pensar que algunas de las respuestas de la asociación libre podrían estar determinadas por otros procesos aparte de los inconscientes. Por ejemplo, siempre es posible mentir (en el sentido de una aceptación consciente de falsedad). ¿Deberían contar las mentiras voluntarias como muestras del inconsciente? Si es así, ¿hasta que punto?

En el caso "Dora", encontramos otro ejemplo de la visión de Freud sobre la inevitabilidad del determinismo inconsciente. Después de negar la sugerencia de Freud de que ella se habría enamorado de su padre, le contó a Freud la historia de una niña que quería que su madre muriera para que pudiera casarse con su padre. Freud anotó lo siguiente:

Tengo el hábito de considerar las asociaciones tales como ésta, que trae al frente algo que está de acuerdo con una aseveración mía, como una confirmación del inconsciente de lo que he dicho. Ningún otro tipo de "Sî" puede ser extraído del inconsciente; no hay tal cosa como un inconsciente "No" (O.C., VII, p. 57)

A este comentario añade en 1923 una nota a pie de página que aclara el punto que estoy discutiendo:

Hay otra forma muy notable y enteramente fiable de confirmación del inconsciente, que no había reconocido cuando esto fue escrito: a saber, una explicación de la parte del paciente de "No pensé eso" o "No pensé en eso". Esto puede ser traducido directamente a: "Sí, yo era inconsciente de eso" (ídem).

Sí significa sí, y no, ¡isí tambien! En sus escritos posteriores Freud reconoció el problema de la infalibilidad cuando caracterizaba la situación con el dicho: "Cara, yo gano, sello tu pierdes". Aun cuando trató de minimizar el enfoque dogmático de los datos ${ }^{15}$, queda la sospecha de que en la recolección de datos, hay pocas esperanzas de desconfirmación de datos.

15 En Negación, muestra su preocupación acerca del significado de "no". Más que un simple desacuerdo con el analista, un "no" puede significar que el paciente quiere reprimir el contenido de una idea en el inconsciente. Negar un pensamiento es equivalente a decir: "Esto es algo que preferiría reprimir” (O.C., XIX, pp. 235-236). 
Las asociaciones libres parecen cuestionar el mismo fundamento del psicoanálisis, el determinismo, puesto que ¿cómo podemos dar cuenta de la diferencia entre determinantes y asociaciones? Cuando se le pregunta a un paciente por una serie de asociaciones, ¿cómo puede el analista seleccionar las respuestas como resultado de las causas de la neurosis de las asociaciones con cualquier cosa? Freud llegó tan lejos como sugerir que aún las asociaciones con números escogidos al azar podrían conducir a las ideas que causaban los síntomas neuróticos (O.C., VI, pp. 250-251). Un hecho que es común es que los analistas influyen en el tipo de asociaciones que terminan produciendo sus pacientes. Y, no debería sorprender, sus asociaciones tienen una semejanza muy cercana a la postura teórica de los analistas (Eagle, 1983, p. 38). Como Zubin comenta irónicamente: En las asociaciones libres, los pacientes freudianos informan tener sueños freudianos, los pacientes jungianos, sueños jungianos, y los pobres pacientes rogerianos, $¡$ no tienen sueño alguno! (Zubin, citado en Macmillan, 1997, p. 584). En algunos pasajes, Freud no tenía mucho reparo en crear datos en nombre del beneficio terapéutico. En el caso del "Pequeño Hans", leemos:

En un psico-análisis, el médico siempre da a su paciente (algunas veces en mayor y en algunas veces en menor grado) las ideas anticipatorias cons- cientes con cuya ayuda él [paciente] puede estar en la capacidad de reconocer y captar el material inconsciente. Puesto que hay pacientes que necesitan más de tal asistencia y otros que necesitan menos (O.C., X, pp. 104-105).

Pero, nuevamente, debido a su determinismo Freud tiene la confianza de que tal intervención lleva inevitablemente al inconsciente. Así, dice:

La información que el médico da al paciente se deriva a su vez de la experiencia analítica; y empero, es suficientemente convincente si, aun con el precio de la intervención del médico, nos es permitido descubrir la estructura del material patógeno y simultáneamente disiparlo (ídem.).

Claramente, para Freud la influencia de las asociaciones por parte del analista quedan descartadas. Pero esto último no puede ser probado, es simplemente un dogma.

Se podría replicar que en la ciencia, las observaciones nunca están libres de la influencia de una teoría, por lo tanto, uno esperaría que los datos estén coloreados por las convicciones teoréticas del analista. En un sentido, esto parece ser cierto. Se diseñan experimentos para probar las ideas de uno. Pero hay una distinción importante. A diferencia del psicoanálisis, en otros ejemplos en la ciencia hay un esfuerzo por presentar los datos de un modo tal que pueda haber acuerdo en algo tan básico como el hecho de que un fenómeno realmente ocurrió. Consideremos la medición del desplazamiento de las estrellas durante un eclipse como prueba de la relativi- 
dad general. La medición hecha en 1919 debía falsear la teoría gravitacional de Newton. Pero los datos eran suficientemente transparentes como para probar o no la teoría: de acuerdo a Einstein, las estrellas debían estar desplazadas el doble de lo predicho por la mecánica newtoniana. Los datos se presentaron de tal manera que podrían haber sido recolectados por cualquiera independientemente de sus convicciones sobre cualquiera de las dos teorías en juego. Aun cuando las observaciones no fueran de ninguna manera ideales ${ }^{16}$, éstas favorecieron las expectativas relativistas. Y los datos subsiguientes, tales como la observación del efecto del corrimiento al rojo de la luz proveniente del sol, reforzaron las primeras observaciones. En el caso del psicoanálisis, en contraste, los datos posteriores no parecen cuestionar ninguno de los anteriores. Parte de la explicación se debe a la opacidad de los datos que estimulan cualquier interpretación (Eagle, 1983,

16 Las observaciones produjeron una serie de placas que se consideraron inutilizables. Sólo una parte de los datos fueron finalmente usados para las interpretaciones. La razón fue que, debido a las condiciones climatológicas, algunas de las placas no mostraron las estrellas que se estaban buscando. Es verdad que hay un proceso discriminatorio en todas las observaciones, pero está motivado para disminuir la ambigüedad. Para mayores referencias del problema de la medición del desplazamiento de las estrellas véase Earman \& Glymour (1980). p. 40). Y otra parte es que no hay forma de verificar los datos independientemente de la persuasión teórica. En este sentido, no podrían haber experimentos doble-ciego en un entorno psicoanalítico, puesto que para recolectar los datos, uno debe presuponer la hipótesis que se quiere probar. Y, para repetir lo que he dicho anteriormente, sería muy difícil determinar si los datos están proviniendo del paciente o si están siendo generados por el analista a través de su influencia.

\section{INTERPRETACIÓN DE LOS DATOS}

Como vimos anteriormente, el psicoanálisis depende de la interpretación, tanto para explicar la causa de las neurosis como para los efectos terapéuticos. Algunos psicoanalistas consideran que la interpretación es aún más importante que los datos que se observan. En esta sección me ocuparé del problema general de la validez de la interpretación.

Interpretación aquí se refiere al uso que hacen los psicoanalistas de los datos traídos de la asociación libre. Asumiendo el modelo de la psicología popular de creencias y deseos, el psicoanalista interpreta los datos para develar el contenido -el llamado contenido latente- que a su vez causa la dolencia del paciente. Al develar el contenido de los deseos inconscientes, los analistas esperan llenar los vacíos arriba mencionados. Esto es lo que, de acuerdo con el psicoanálisis, da inteli- 
gibilidad o significación al fenómeno mental que, de otro modo, no tendría sentido.

\section{Validación de las interpretaciones}

Una nota que ha caracterizado el psicoanálisis desde el principio ha sido la falta de reglas metodológicas para la recolección y procesamiento de los datos. El mismo Freud se opuso a una aproximación de tipo libro de texto para la interpretación, y se fiaba más en la experiencia del analista. Y esa aproximación es básicamente la misma en la actualidad. Considérese lo que ocurre en el proceso de la asociación libre. A pedido del analista, el paciente comienza a nombrar todas las ideas que se le vienen a la conciencia, digamos de sus sueños. El analista -supuestamente manteniendo una atención suspendida de opinión- debe tratar de juntar las piezas y construir una historia que eventualmente deberá llenar los vacíos causales. Pero, ¿cómo debería proceder el analista cuando está interpretando? ¿Deberá parar el flujo de datos o deberá dejar que fluya indefinidamente? Seguramente debe haber una diferencia en la interpretación si es que hay más o menos elementos para unir. Se podría argüir que algunas asociaciones son irrelevantes o redundantes. Pero eso sería una cuestión de gusto personal en el mejor de los casos. Puesto que, si se es consistente con el determinismo, nada de lo que el paciente produce es azaroso, entonces, ¿cuántos datos se necesitan para producir una interpretación válida? Esto último es muy importante porque nada impide que hechos y sentimientos expresados por el paciente sean ignorados por el analista. Como dice Glymour, sin reglas, el intérprete "tiene en su poder... obtener asociaciones que pueden caber en una historia plausible de cualquier tipo específico que quiera tener el intérprete" (1983, p. 62). Por lo tanto, no habría criterios para evaluar la validez de interpretaciones en competencia. Varios estudios parecen confirmar esta conclusión.

Lakin y Lebovits encontraron que comparados con otros psicoterapeutas (eclécticos y centrados en el cliente), los psicoanalistas interpretan hechos aislados de sus pacientes de manera más arbitraria. Para los analistas, pocos hechos eran necesarios para generar diferentes interpretaciones, al punto que habían algunas interpretaciones diametralmente opuestas. De hecho, los psicoanalistas eran más propensos a tolerar asociaciones mutuamente contradictorias y menos preocupados por la posibilidad de equivocarse. Más aún, en lo que respecta a la etiología, los analistas estaban en desacuerdo acerca de los factores causales, y la especulación era más la norma que la excepción: para uno era "deprivación temprana", para otro, "una madre hostil y rechazante", y para otro, era "un padre distante e indiferente" (Fisher \& Greenberg, 1978, pp. 390-396). Uno 
podría explicar tales diferencias en la falta de entrenamiento y práctica del intérprete, como algunas veces ocurre en la ciencia médica. Sin embargo, recientes estudios prueban todo lo contrario. Por ejemplo, Cutler et al. compararon psicoanalistas experimentados y poco experimentados y encontraron que había mayor acuerdo en las intepretaciones hechas por el grupo con poca experiencia (ibíd., pp. 397407). Si fuéramos a juzgar el psicoanálisis desde un modelo médico, la disparidad en la evaluación de los factores etiológicos, ¿contribuiría en algún sentido a la mayor comprensión de la enfermedad? Aun cuando asumiéramos que la búsqueda del mayor factor causal involucrado en el desarrollo de una enfermedad no es una tarea fácil, no parece razonable endosar una metodología que invita al desacuerdo en cosas básicas. Además, no existen guías para diferenciar entre interpretaciones válidas e inválidas, no hay forma de decir cuáles interpretaciones están en el camino correcto y cuáles fuera de lugar. Si no hay una forma de identificar una interpretación correcta, el veredicto final es dejado en manos del practicante. Esto sólo puede llevar a la creencia de que hay algunos "expertos" que no fallan. El problema es que en la pseudociencia es conocida la habilidad de muchos "expertos". Hay numerosos ejemplos en la historia de la ciencia que atestiguan la presencia de algunos con "manos de oro", cuyos experimentos funcionaban sólo con ellos. Estos casos resultan frecuentemente en fraude ${ }^{17}$.

Entonces, ¿por qué no dejar la validación de la interpretación en manos del paciente? Despúes de todo, el paciente debería ser capaz de reconocer los eventos interpretados como algo que él ha experimentado. La confirmación o no confirmación podría ser deducida o bien del reporte verbal o bien del comportamiento del paciente. Pero esto tampoco funcionaría, porque nos encontramos una vez más en el círculo de confirmación que el analista controla. Si el paciente confirma la interpretación bien podría tomarse como un mecanismo de defensa, digamos, conformidad con el analista. Si el paciente rechaza la interpretación puede ser tomada como una interpretación falsa o como una resistencia a revelar los contenidos de su inconsciente. Esta incertidumbre nos lleva al siguiente problema relacionado.

\section{El problema de la verdad en el psicoanálisis}

Cuando un analista interpreta datos uno presume que lo que está haciendo es revelando lo que realmente ocurrió en la vida del paciente. Intuitivamente se asumiría que para que algo cause una enfermedad, debe ser algo real. Por

17 Cf. Collins y Pinch (1993, Cap.1). 
lo tanto, se esperaría que la interpretación debería proporcionar un informe de los acontecimientos como realmente ocurrieron.

Este modo de visualizar la tarea de un analista es considerado por algunos críticos como una forma de "realismo ingenuo" (Hanly, 1990, p. 375). Los críticos del realismo ingenuo preferirían no tener un compromiso de verosimilitud entre la interpretación y aquello que ha causado la neurosis del paciente. En cambio, sostienen que la reconstrucción psicoanalítica de la vida del paciente no tiene por qué ser verdadera. Lo que cuenta como una buena interpretación -y no una interpretación verdadera- es su coherencia, su consistencia interna y la inteligibilidad narrativa. De acuerdo con esta perspectiva, el psicoanálisis no tiene nada que ver con explicaciones causales sino con la construcción de narrativas. Como se puede inferir, las narrativas no corresponden a nada, y son juzgadas, quizás, por su persuasión o por su belleza interna.

La motivación detrás de esta perspectiva es comprensible. Parte de la razón estriba en que se puede obtener beneficios terapéuticos en ausencia de una búsqueda causal. Pero el éxito terapéutico puede deberse a otros factores. Otra razón para cuestionar la verosimilitud es el hecho de que para cualquier persona lo real es cómo un evento es sentido y cómo es percibido. Puede darse el caso de que un evento no tuvo lugar en el mundo y, sin embargo, una persona puede creer que ocurrió. Y esta situación podría constituir un patógeno. Por tanto, es importante especificar la noción de evento psicológico, especialmente cuando éste conduce a un comportamiento neurótico ${ }^{18}$. Pero pensar en eventos mentales en una forma diferente no justifica el abandono de la búsqueda de las causas reales. Si disolvemos el problema de la verosimilitud, cualquier historia es igualmente aceptable por derecho propio. Aun cuando ha habido esfuerzos por tener criterios para juzgar narrativas, no habría forma de distinguir entre la explicación y la persuasión ${ }^{19}$. Y esto tiene una consecuencia práctica. Las narrativas pueden ser tan flexibles que pueden acomodar cualquier interpretación de datos, y, de ese modo, confirmar principios que son considerados

18 Este pensamiento está fundamentado en la crítica que hace Nash de la concepción de evento mental de Grünbaum. Grünbaum considera que un evento que nunca tuvo lugar no podría ser un patógeno. Y parece que cuando se refiere a eventos tiene en mente el mundo externo. Nash, creo que correctamente, nos indica que "un patógeno puede ser una idea". Preguntarnos si las ideas realmente ocurren es una pérdida de tiempo, dice Nash, porque parece que, a veces, causan un montón de problemas (Nash, 1989, pp. 334-335).

19 Spence ha tratado de proporcionar tales criterios. Pero su "criterio estético de verdad" es solamente eso, una apreciación de la belleza de la reconstrucción que evade ser verdadera o falsa. Cf. Roth (1991, p.183). 
contradictorios por diferentes psicoanalistas. Y, correlativamente, si no hay forma de juzgar narrativas -aparte de la vaguedad de la inteligibilidad- se vuelven inmunes a la crítica.

Cuando se deja la pregunta de la verosimilitud, no hay una búsqueda de por lo menos una aproximación de lo que realmente ocurre en la vida de una persona. Y esto último importa porque hay varias consecuencias. Primero, como una ciencia médica, el psicoanálisis debería explicar qué es lo que causa una enfermedad, no sólo por razones terapéuticas, sino también por razones preventivas. Conociendo las causas de un comportamiento no deseado, podemos tomar medidas para prevenir que ese comportamiento ocurra. Pero, si las explicaciones supuestas admiten elementos de ficción, no parece haber una comprensión real de lo que tiene lugar en una persona. Segundo, el desarrollo de una teoría tiene que estar basado en hechos que puedan ser de alguna manera empíricamente probados.

Cuando Freud interpretó el sueño del "Hombre Lobo" lo hacía bajo la presuposición de que el paciente en una edad temprana de hecho fue testigo de que sus padres tenían relaciones sexuales. La conexión entre sus padres teniendo sexo y su sueño con lobos no era accidental. Al contrario, el "Hombre Lobo" buscó ayuda porque tenía una fobia por los lobos, y esa fobia, de acuerdo con Freud, era debido al temor que tenía el paciente por su padre. En su sueño, su padre había sido reemplazado por el lobo, que estaba en una posición erecta, asemejando a su padre cuando estaba teniendo relaciones sexuales. Para Freud era claro que la escena sexual tenía un efecto patogénico y afectó el desarrollo sexual del "Hombre Lobo" (O.C., 17, pp. 29-47). Presumiblemente, la teoría psicoanalítica del desarrollo sexual y de los deseos conflictivos de contenido sexual deben haber tenido origen en la experiencia clínica de Freud. Si las interpretaciones no hubieran tenido semejanza con lo que realmente ocurrió, no habrían tenido propósito alguno en la construcción de la teoría.

No sostengo que la pregunta por la verosimilitud sea fácil, especialmente cuando nos referimos a los procesos inconscientes. Pero, queda en el psicoanálisis el peso de la justificación de la explicación de esos procesos. La verificación de tales instancias obviamente exige un esfuerzo en la búsqueda de causas. Pero si no hace el esfuerzo, las explicaciones que pueda ofrecer el psicoanálisis serán meramente historias que incluyen ilusiones y negaciones de la verdad. Podrán ser historias muy coherentes, pero, como aprendemos en lógica, así como un argumento puede ser válido pero falso, una narrativa puede ser coherente pero falsa también.

Algunos psicoanalistas han tratado de ofrecer una visión más realista y causal 
de la explicación ${ }^{20}$. Pero estos esfuerzos se enfrentan a métodos que en último término no están comprometidos con un enfoque causal y, por lo tanto, están destinados a fracasar.

\section{Interpretación y terapia}

Dije anteriormente que en un modelo médico, los resultados terapéuticos no necesariamente cuestionan los informes etiológicos. En el psicoanálisis, sin embargo, hay una estrecha relación entre la teoría y la terapia. La interpretación no es un fin en sí mismo cuando establece el significado, sino que tiene la función de poner al frente lo inconsciente para producir un efecto terapéutico. En este sentido, la efectividad de la terapia psicoanalítica tiene una relación con la validez de la interpretación. El problema es que parece no haber una relación entre una interpretación correcta y el resultado de la terapia. La interpretación sólo tiene un valor instrumental.

20 Uno de tales esfuerzos es representado por Edelson, quien respondiendo a Grünbaum reconoce varios problemas a los que se enfrenta el psicoanálisis al tratar de dar una explicación causal y sugiere que el método debe ser revisado: "Grünbaum tiene toda la razón... de mantenerse escéptico a la realización de tal programa o que si se toma esa dirección se corrija algún día los defectos en los fundamentos del psicoanálisis que él ha identificado. Aquí, por supuesto, sólo el tiempo lo dirá. Pero, a mis colegas, les sugiero que empecemos con esperanza" (1988, pp. 276277).
En los últimos años Adolf Grünbaum ha venido cuestionando la terapia psicoanalítica basado en una supuesta postura de Freud. De acuerdo a Grünbaum, Freud está comprometido con la proposición de que "la correcta comprensión por parte del analizado de la etiología de su aflicción y de la dinámica inconsciente de su carácter es, a su vez, causalmente necesario para la conquista terapéutica de su neurosis" (1984, pp. 139-140). Más tarde, Grünbaum identifica un pasaje en el que Freud sostiene que "los conflictos [del paciente] sólo serán resueltos y sus resistencias vencidas si las ideas anticipatorias que se le dan corresponden con lo que es real en él" (1984, p. 138). Grünbaum llama a este razonamiento el "argumento de correspondencia"21. Si Freud se suscribió a este argumento o no, es asunto que compete a los eruditos, pero parece razonable preguntarnos si debería haber una conexión entre una buena interpretación y un resultado terapéutico exitoso. En el núcleo de la demanda de Grünbaum está la intuición de que una interpretación que está diseñada para calzar con el paciente y sus circunstancias debería ser más beneficiosa que una interpretación que no lo fuera. Es obvio que los psicoanalistas no pueden ofrecer la promesa de la felicidad, y que la concientización producto de una inter-

21 El nombre en el original inglés es "tally argument". 
pretación correcta no puede garantizar siempre una cura. Pero, ciertamente, no podríamos esperar que cualquier interpretación diera el mismo resultado. $\mathrm{Si}$ así fuera, entonces otros factores deberían ser responsables del resultado. Es por esto que Freud, preocupado por la contaminación de la sugestión, pensó que las curas que no eran resultado de una interpretación correcta no serían permanentes y que los conflictos subyacentes y no resueltos conducirían al desarrollo de nuevos síntomas (Fisher \& Greenberg, 1996, p. 219). Sin embargo, los resultados empíricos sugieren que los contenidos de las interpretaciones no están estrictamente ligados al resultado terapéutico ${ }^{22}$.

De acuerdo con el "argumento de la correspondencia", esperaríamos que una interpretación fidedigna debería resultar, al menos, en una reducción de la ansiedad. La evidencia indica lo contrario. Interpretaciones falsas o ambiguas que pueden aplicarse a cualquier personalidad son aceptadas entusiastamente por individuos que creen que son específicamente exactas para ellos

22 Aun cuando la historia de la confirmación empírica del psicoanálisis ha estado caracterizada por la resistencia de los psicoanalistas para poner a prueba sus teorías y terapias, en los últimos años ha habido un esfuerzo por estudiar y comparar lo que ocurre en los consultorios psicoanalíticos. Debo enfatizar que algunos estudios son todavía preliminares y se necesita incorporar más refinamientos metodológicos. Sin embargo, algunos hechos pueden permitir algunas conclusiones. Al menos tentativamente.
(Fisher \& Greenberg, 1996, p. 239). Aún más, este efecto impredecible puede ser usado para provocar un cambio conductual deseable. Algunos radicalmente sostienen que las interpretaciones que no tienen relación con los datos proporcionados por los pacientes pueden, sin embargo, producir un resultado positivo. En 1964 Mendel manifestó que interpretaciones intencionalmente inexactas podían influenciar en el comportamiento (ibíd., p. 240).

Estos estudios no han considerado un seguimiento de los pacientes después de la recuperación, que podría ayudar a medir el asunto de la sugestión que tanto preocupaba a Freud. Sin embargo, no sólo no parece tarea fácil poder aislar la sugestión, sino que ocurre que la sugestión juega un rol importante si se toma como un medio para el éxito terapéutico. Pronto el analista se convierte en persuasor, $y$, como nos recuerdan Fisher y Greenberg: "la sustitución de 'esto es lo que le ha pasado a usted' por 'esto es lo que realmente pasó' es un reconocimiento abierto de que el tratamiento descansa sobre un fuerte elemento sugestivo" (1996, p. 242). En tal visión, se deja de lado la búsqueda de causas reales, se descalifica los datos obtenidos en el entorno clínico y nos hace cuestionar lo extraña que parece ser esta ciencia clínica. Pero, y no debería sorprender, estas evidencias tienen eco en la voz de los hermenéuticos, quienes habiéndose rendido a la pregunta de la verosimilitud, abrazan 
entusiastamente el valor instrumental de la terapia psicoanalítica. En su exposición, Sherwood dice:

Los beneficios terapéuticos... resultarán de la aceptación por parte del paciente de la narrativa psicoanalítica, sea que la narrativa coincida o no con la causa real del comportamiento neurótico del paciente. La eficacia terapéutica, entonces, puede no guardar relación alguna con la verdad de tales narrativas (1969, pp. 250-251).

Los propulsores de esta visión de la terapia deben demostrar cómo funciona entonces la terapia, si es que funciona. No ayuda solamente reconocer su eficacia, aun cuando fuera cierta. Si ése fuera el caso, entonces deberíamos unirnos al Freud tardío cuando disocia la verdad del éxito terapéutico y afirma:

"Yo creo que nuestras curas pueden competir con aquellas de Lourdes. Hay muchas más personas que creen en los milagros de la Santísima Virgen que en la existencia del inconsciente" (O.C., 15 , p. 152).

La eficacia de la terapia psicoanalítica debido a su singular método interpretativo no parece tampoco tan clara. Una pregunta pertinente es saber cómo la terapia psicoanalítica se compara en efectividad con otras formas de psicoterapia. Varios estudios sugieren que no hay evidencia de que con el psicoanálisis se obtienen mejores resultados que con tratamientos con otra etiqueta.

Algunas décadas atrás Heine comparó resultados terapéuticos de tres diferentes tendencias: psicoanalítica, adleriana y no-directiva. Concluyó que no había diferencias significativas en los cambios atribuibles a la membresía teórica del terapeuta. Señaló que más que las características particulares de cada teoría, era el agente terapéutico el responsable por el resultado (Heine, 1953, pp. 16-23). Los estudios de Heine se han visto corroborados por otros posteriores. Estos últimos han tenido más rigurosos controles de los tratamientos para una mejor evaluación. Fisher y Greenberg (1996, pp. 207211) han analizado las investigaciones recientes (Svartberg \& Stiles, 1991; Crits-Christoph, 1992; Luborsky et al., 1993) y concluyen lo siguiente:

1. Una terapia psicoanalítica breve es mejor que ninguna.

2. Comparando el tratamiento psicoanalítico con otros tipos de tratamientos psicológicos y psiquiátri$\cos$, no se observa ventaja alguna en ninguno de los enfoques.

Como podemos ver, no hay monopolio en la efectividad del tratamiento. Estos resultados sugieren que en todas las formas de tratamiento se pasan por alto factores comunes que son responsables de los resultados. Algunos sugieren que uno de esos factores pueda ser la relación de cuidado entre la sólida figura del terapeuta y el paciente que necesita desarrollar nuevas formas de verse a sí mismo y a sus problemas (Fisher \& Greenberg, 1996, p. 212). Esto es consistente con un estudio de 
Piper y su equipo, quienes analizaron el efecto de un número de interpretaciones que involucraban transferencia. Al contrario de lo que el psicoanálisis hubiera predicho, y para sorpresa de Piper y sus colegas, el estudio mostró que había una relación inversa entre el número de interpretaciones y el resultado terapéutico. La explicación que Piper et al. dieron a los datos inesperados era que un alto uso de la transferencia se traducía en el paciente en la sensación de sentirse criticado o en el esfuerzo equivocado del analista al tratar de vencer la resistencia del paciente (ibíd., p. 233). Estos resultados sugerirían que el paciente percibía que la relación de cuidado estaba siendo amenazada por la hipercrítica del terapeuta o por la impaciencia mostrada por el terapeuta al proporcionar más interpretaciones en su lucha por obtener algunos resultados.

La responsabilidad de demostrar los beneficios del psicoanálisis queda en manos de los que defienden tal pretensión. El breve recuento de los estudios recientes muestra, sin embargo, que no parecen haber beneficios especiales que se obtengan gracias a la terapia psicoanalítica si la comparamos con otros enfoques y la razón de los beneficios manifestados pueden deberse a otros factores fuera del proceso interpretativo.

Finalmente, un comentario adicional. Algunos sostienen que porque no hay una mejor teoría que trate de los deseos y creencias inconscientes, deberíamos quedarnos con el método clínico del psicoanálisis (Levy, 1996, p. 165). Primero, como he querido demostrar, no hay mucho que recomendar del psicoanálisis. Las deficiencias metodológicas cuestionan la validez de sus enunciados. Segundo, asumir que el psicoanálisis es el único enfoque posible para el inconsciente es sencillamente falso. Están emergiendo nuevas teorías y éstas están propugnando un mayor rigor en su método. Aquí se puede mencionar la teoría del "inconsciente como congruencia interna" (Orbach, 1996, Cap. 6). Esta teoría todavía usa los vocablos populares pero de una manera que pueden ser mejor examinados y aplicados.

\section{ConClusión}

El psicoanálisis, tal como es visto por sus practicantes, puede ser descrito como una ciencia médica, cuya meta es la identificación y tratamientos de las neurosis. Además, el psicoanálisis puede ser visto como una extensión de la psicología popular puesto que postula la existencia de creencias y deseos, al menos para el reino inconsciente. En forma análoga a la psicología popular, el psicoanálisis explica las interacciones causales de las creencias y deseos inconscientes. Las explicaciones que genera tienen el propósito de llenar los vacíos de las conexiones causales entre diferentes contenidos mentales. Se sostiene que en cualquier 
ciencia médica debería haber un compromiso con una investigación realista acerca de las causas de una enfermedad. El psicoanálisis, sin embargo, no parece satisfacer tal compromiso. Se argumenta cómo el modo en que el psicoanálisis recoge los datos sugiere varios problemas que finalmente conducen a la arbitrariedad. Los datos en el psicoanálisis son tan oscuros que virtualmente se promueve la posibilidad para cualquier interpretación. De tales datos es difícil ver cómo se puedan derivar constructos teóricos. En cuanto a la interpretación de los datos, no hay guías para decidir cuáles interpretaciones son verdaderas o falsas. Eso emana del hecho de que no hay criterios adecuados para la interpretación al carecer de una teoría de verdad. Puesto que no hay ningún compromiso con alguna forma de realismo, las interpretaciones tienen un valor instrumental para los resultados terapéuticos. El análisis de la relación entre la interpretación y la terapia muestra que, en esencia, los psicoanalistas son instrumentalistas en cuanto a la explicación: las explicaciones son buenas en tanto inducen al beneficio terapéutico. Así, el psicoanálisis no está comprometido con una visión realista de la psicología popular.

\section{REFERENCIAS}

Bateman, A. \& Holmes, J. (1995). Introduction to psychoanalysis: contemporary theory and practice. London: Routledge.

Broncano, F. (Ed.) (1995). Enciclopedia Iberoamericana de Filosofía. La mente humana (Vol. 8) Madrid: Trotta.

Cohen, R. \& Laudan, H. (Eds.) (1983). Physics, philosophy and psychoanalysis. Dordrecht: Reidel Publishing.

Collins H. \& Pinch, T. (1993). The golem: what everyone should know about science. Cambridge: Cambridge University Press.

Eagle, M. (1983). The epistemological status of recent developments in psychoanalytic theory. En R. Cohen y $\mathrm{H}$. Laudan (Eds.), Physics, philosophy and psychoanalysis. Dordrecht: Reidel Publishing.

Earman, J. \& Glymour, C. (1980). Relativity and eclipses: The British Eclipse Expeditions of 1919 and their predecessors. Historical Studies in the Physical Sciences, 11, 49-85.

Edelson, M. (1988). Psychoanalysis: a theory in crisis. Chicago: University of Chicago Press.

Fisher, S. \& Greenberg, R. (1978). The scientific evaluation of Freud's theories and therapy. Hassocks, Sussex: The Harvester Press.

Fisher, S. (1996). Freud scientifically reapraissed: Testing the theories and therapy. New York: John Wiley \& Sons.

Freud, S. (1953-1974). The Standard Edition of the Complete Psychological 
Works of Sigmund Freud. London: The Hogarth Press and The Institute of Psycho-Analysis.

Glymour, C. (1983). The theory of your dreams. En R. Cohen y H. Laudan (Eds.), Physics, philosophy and psychoanalysis. Dordrecht: Reidel $\mathrm{Pu}-$ blishing.

Grünbaum, A. (1984). The foundations of psychoanalysis: A philosophical critique. Berkeley: University of California Press.

. (1992). Validation in the clinical theory of psychoanalysis: A study in the philosophy of psychoanalysis. Madison, CT: International Universities Press.

Hanly, C. (1990). The concept of truth in psychoanalysis. International Journal of Psychoanalysis, 71, 375-383.

Heine, R.W. (1953). A comparison of patient's reports on psychotherapeutic experience with psychoanalytic, nondirective and adlerian therapists. American Journal of Psychotherapy, 7, 1623.

Hopkins, J. (1991). The interpretation of dreams. En J. Neu (Ed.), Cambridge companion to Freud. Cambridge: Cambridge University Press.

Klein, G. S. (1976). Psychoanalytic theory: An exploration of essentials. New York: International Universities Press.

Levy, D. (1996). Freud among the philosophers: the psychoanalytic unconscious and its philosophical critics. New Haven: Yale University Press.

Macmillan, M. (1997). Freud evaluated: the completed arc. Cambridge, MA: The MIT Press.
Miller, R. (1988). A clinical science. Canadian Journal of Philosophy, 18, 659-80.

Nash, M. (1989). Grünbaum and psychoanalysis. Philosophical Psychology, 2, 325-343.

Neu, J.(1991). (Ed.). Cambridge companion to Freud. Cambridge: Cambridge University Press.

Orbach, I. (1995). The hidden mind: Psychology, psychotherapy and unconscious processes. Chichester, Sussex: John Wiley \& Sons.

Rangell, L. (1988). The future of psychoanalysis: The scientific crossroads. Psychoanalysis Quarterly, 57, 313-340.

Richardson, R. (1990). The "Tally Argument" and the validation of psychoanalysis. Philosophy of Science, 57, 668-676.

Roth, P. (1991a). Interpretation as explanation. En D. Hiley, J. Bohman, \& R. Shusterman (Eds.), The interpretative turn: Philosophy, science, culture. Ithaca: Cornell University Press.

(1991b.). Truth in interpretation: The case of psychoanalysis. Philosophy of the Social Sciences, 21,175-195.

Rustin, M. (1987). Psychoanalysis, philosophical realism, and the new sociology of science. Free Associations, 9, 102136.

Sachs, D. (1991). In fairness to Freud: A critical notice of The foundations of psychoanalysis by Adolf Grünbaum. En J. Neu (Ed.), Cambridge companion to Freud. Cambridge: Cambridge University Press.

Schafer, R. (1976). A new language for psychoanalysis. New Haven: Yale University Press. 
Schwaber, E. A. (1990). Interpretation and the therapeutic action of psychoanalysis. International Journal of Psychoanalysis, 71, 229-240.

Sherwood, M. (1969). The logic of explanation in psychoanalysis. New York: Academic Press.

Wallerstein, R. (1988). One psychoanalysis or many? International Journal of Psychoanalysis, 69, 5-21.

(1990). Psychoanalysis: The common ground. International Journal of Psychoanalysis, 71, 3-20.

Wollheim, R. (1993). The mind and its depths. 